Questions vives

\section{Questions Vives}

Recherches en éducation

$N^{\circ} 29 \mid 2018$

La bienveillance en éducation : approches compréhensives et critiques

\title{
«Conforter une école bienveillante et exigeante » : représentations, préoccupations et pratiques déclarées
}

"Reinforce a caring and demanding school": representations, concerns and selfreported practice

\section{Éric Saillot}

\section{Q OpenEdition}

\section{Journals}

Édition électronique

URL : http://journals.openedition.org/questionsvives/3280

DOI : 10.4000/questionsvives.3280

ISSN : 1775-433X

Éditeur

Université Aix-Marseille (AMU)

Édition imprimée

ISBN : 978-2-912643-53-7

ISSN : 1635-4079

Référence électronique

Éric Saillot, « «Conforter une école bienveillante et exigeante » : représentations, préoccupations et pratiques déclarées », Questions Vives [En ligne], № 29 | 2018, mis en ligne le 19 décembre 2018, consulté le 19 avril 2019. URL : http://journals.openedition.org/questionsvives/3280 ; DOI : 10.4000/ questionsvives.3280

Ce document a été généré automatiquement le 19 avril 2019.

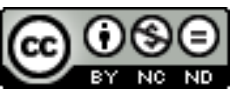

Questions Vives est mis à disposition selon les termes de la licence Creative Commons Attribution Pas d'Utilisation Commerciale - Pas de Modification 4.0 International. 


\title{
«Conforter une école bienveillante et exigeante » : représentations, préoccupations et pratiques déclarées
}

\author{
"Reinforce a caring and demanding school": representations, concerns and self- \\ reported practice
}

Éric Saillot

Dans le cadre de la Loi de refondation de l'école ${ }^{1}$ de 2013, la notion de bienveillance est apparue dans les prescriptions officielles, notamment dans le référentiel relatif au renforcement de l'éducation prioritaire (Axe 2 : Conforter une école bienveillante et exigeante) ou encore dans le guide élaboré pour améliorer le climat scolaire : Une école bienveillante face aux situations de mal-être des élèves (Éduscol, 2013). Le Conseil national de l'innovation pour la réussite éducative (Lapeyronnie, 2014) présente la bienveillance comme «la condition nécessaire à l'engagement mais aussi à l'efficacité de l'école» (p. 25). Loin de penser que les enseignants ont attendu ces prescriptions à la bienveillance pour la mettre en œuvre, nous avons souhaité comprendre leurs représentations, leurs préoccupations et les pratiques déclarées qu'ils associent à cette notion nouvellement institutionnalisée. Nous empruntons le terme préoccupations des enseignants à Bucheton et Soulé (2009) qui les qualifient d'« enchâssées » dans leur modèle d'analyse du multi-agenda. Pour Faïta et Saujat (2010), les préoccupations expriment ce que les professionnels voudraient faire à travers leurs «occupations" (ce qu'ils font effectivement en situation de travail), d'où l'expression employée par Curie et Dupuy (1996) qui parlent de « pré-occupations ». Notre problématique est donc avant tout descriptive et compréhensive mais également critique, à propos de cette nouvelle prescription qui vise à «conforter une école bienveillante et exigeante ». Comment les professionnels des équipes pédagogiques et éducatives ont-ils appréhendé ces injonctions à la bienveillance? Quelles sont les représentations de la bienveillance en éducation chez les professionnels de l'école? Quelles préoccupations professionnelles pensent-ils avoir pour «conforter une école bienveillante et 
exigeante » ? Pour répondre à ce type de questionnements, nous avons mené une enquête dans l'académie de Caen en partenariat avec le rectorat, notamment les inspecteurs pédagogiques régionaux responsables de la mission climat scolaire. Dans une première partie, l'approche ergonomique (Amigues, 2009) nous amène à étudier les prescriptions de l'Éducation nationale en ce qui concerne la bienveillance à l'école. Ensuite, nous présenterons les contours méthodologiques de cette enquête: le questionnaire, l'échantillon visé, les réponses obtenues. Nous présenterons finalement les principaux résultats de cette enquête sur la notion de bienveillance à l'école, en matière de représentations, de préoccupations et de pratiques déclarées, avant d'analyser dans une partie discussion les principales tensions et ambiguités.

\section{La notion d'école bienveillante : une émergence institutionnelle récente}

\subsection{La bienveillance placée au cœur de l'innovation pour la réussite éducative}

Dans la synthèse des travaux du Conseil national de l'innovation pour la réussite éducative (2014) dirigé par le sociologue Didier Lapeyronnie, la bienveillance est au cœur de la problématique de l'engagement des professionnels des équipes éducatives afin d'améliorer l'efficacité de l'école. Ces professionnels ont souhaité prendre au sérieux les " témoignages récurrents des élèves sur le sentiment d'avoir été à telle ou telle occasion "humiliés" par l'École ou les enseignants» (p. 25) qui constituent l'un des facteurs du décrochage scolaire. La bienveillance repose donc d'abord sur l'absence d'humiliation afin de préserver l'estime de soi des élèves, leur confiance en eux, tout comme celle des professionnels, enseignants et éducateurs. Ce rapport considère que la bienveillance est un «état d'esprit ainsi qu'une orientation des pratiques et des comportements " qui s'appuient notamment sur « un mode d'accueil respectant les particularités de chacun et sur un mode d'apprentissage et d'éducation positif » dans l'esprit de l'école inclusive (Gardou, 2012 ; Tremblay, 2012). La pédagogie qui donne un statut constructif à l'erreur est également évoquée. Il est souligné que la bienveillance « doit donc être intériorisée par les acteurs mais aussi faire l'objet d'une organisation et plus généralement d'une politique » (Lapeyronnie, 2014, p. 26). Le travail d'équipe doit notamment être favorisé par des temps de concertation institutionnalisés et des temps de formation conséquents pendant lesquels les professionnels sont accompagnés. Pour cette commission, la bienveillance est plus qu'un climat, elle est une des dimensions fondamentales des pratiques pédagogiques et éducatives, en lien étroit avec les principes éthiques.

\subsection{La bienveillance en lien étroit avec le climat scolaire}

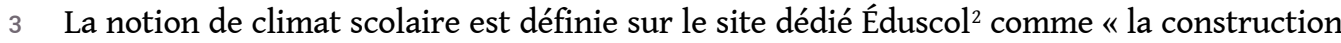
du bien vivre et du bien-être pour les élèves et le personnel de l'école » en un lien étroit avec le «climat de sécurité » et la bienveillance comme le souligne le guide ministériel (2013) à l'attention des équipes éducatives des collèges et des lycées pour prévenir et répondre efficacement aux situations de mal-être des élèves. Ce document institutionnel conseille les équipes sur les comportements considérés comme les plus adaptés, notamment dans les cas d'urgence pour la santé ou dans les situations relevant de la 
protection de l'enfance. Des pistes de réflexion et de travail sont proposées aux équipes éducatives sur le site Eduscol "Climat scolaire» à partir d'une approche systémique autour de sept facteurs : les pratiques partenariales, les stratégies d'équipe, la prévention des violences, la coopération, la co-éducation, le sentiment de justice scolaire, et la qualité de vie à l'école.

\subsection{Les instructions officielles sur l'école bienveillante en éducation prioritaire}

Dans le contexte particulier des réseaux d'éducation prioritaire (REP) dans lesquels les élèves en difficulté en grand nombre ont souvent un sentiment de défiance vis-à-vis de l'école, source de renoncement, d'agressivité ou de violence, l'école bienveillante doit permettre d'apporter un cadre intellectuel, émotionnel et matériel propice aux apprentissages. L'axe 2 de la refondation de l'éducation prioritaire ${ }^{3}$ propose donc de " conforter une école bienveillante et exigeante ", c'est-à-dire une école qui " veille bien » sur les élèves, les sécurise, est attentive à leurs progrès comme à leurs difficultés. Ce texte officiel souligne un point crucial: une école ne peut être bienveillante que si elle est simultanément exigeante et rigoureuse. Trois axes sont proposés pour «conforter une école bienveillante et exigeante »: $1 /$ renforcer les organisations pédagogiques et éducatives, 2/ évaluer les élèves avec bienveillance, 3/ assurer le suivi des élèves.

Le premier point insiste sur la différenciation pédagogique comme réponse à l'hétérogénéité des classes, avec notamment un accompagnement renforcé du travail personnel des élèves. L'observation est présentée comme un geste professionnel fondamental, comme dans nos travaux sur la modélisation d'une posture d'étayage (Saillot, 2015), préalable à tout accompagnement réellement adapté aux besoins spécifiques des élèves, dont les efforts doivent être encouragés et les progrès valorisés. Les projets d'établissements demandent à être recentrés sur les problématiques du climat scolaire qui permet de relier le bien-être des élèves à leurs perspectives de réussite. La continuité école-collège est au cœur du projet de réseau en appui sur le cycle de consolidation grâce au conseil école-collège. La vigilance des équipes éducatives sur les retards et les absences doit être maintenue voire renforcée, tout comme la question cruciale de l'orientation post-troisième, mais également l'accueil adapté pour les enfants de moins de trois ans.

6 Le second point développe les enjeux en matière d'évaluation bienveillante qui doit permettre aux enseignants de réellement considérer l'erreur comme un outil pour enseigner (Astolfi, 1997). Ces instructions officielles appellent à valoriser les progrès et à indiquer des critères de réussite qui permettent d'apporter des conseils précis aux élèves pour comprendre où ils doivent fournir des efforts, contrairement au système classique de la note globale. Des évaluations diagnostiques sont à présent organisées en équipe dès l'entrée dans un nouveau cycle ( $\mathrm{CP} / \mathrm{CM} 1 /$ cinquième) pour ensuite permettre la mise en place de stratégies pédagogiques adaptées aux besoins des élèves : horaires en barrette, co-intervention, programme personnalisé de réussite éducative (PPRE), organisation en groupes de besoins.

7 Le troisième axe souligne l'importance des stratégies collectives dans le suivi des élèves pour prévenir le décrochage scolaire. Un groupe de prévention contre le décrochage scolaire (GPDS) est mis en place dans les établissements, ainsi qu'une commission de suivi des élèves difficiles ou en grande difficulté au sein de chaque école. D’autres coopérations 
entre professionnels sont attendues: un coordonnateur par niveau au collège, la coprésence en classe privilégiée pour l'intervention d'autres personnels (RASED, personnels supplémentaires) et des tutorats mis en place pour les élèves fragiles.

\section{Présentation de l'enquête sur la bienveillance à l'école}

Cette enquête a été réalisée en partenariat avec la mission climat scolaire du rectorat de l'académie de Caen. Cette collaboration nous a permis d'envoyer notre questionnaire à quelque 5200 personnels (enseignants des premier et second degrés, chefs d'établissements, inspecteurs, conseillers principaux d'éducation, infirmières scolaires, assistantes sociales) qui exercent dans les différents établissements scolaires du territoire régional (début 2016). Nous n'avons pas ciblé strictement les enseignants pour ouvrir l'enquête à tous les professionnels de l'école placés sous l'autorité du rectorat, mais nous aurions pu également interroger d'autres professionnels partenaires de l'école et également les parents d'élèves. Notre partenariat avec le rectorat nous a apporté les conditions d'accès à cet échantillon, mais nous avons construit librement notre questionnaire. L'échantillon de départ était représentatif et aléatoire. En effet, c'est le logiciel du rectorat qui a constitué cet échantillon en sélectionnant de façon aléatoire $20 \%$ de l'ensemble des personnels de terrain de l'académie, en respectant la répartition dans chacune des catégories professionnelles. Chaque personnel a été contacté par les adresses mail académiques à trois reprises au début de l'année civile 2016. Ces messages invitaient les professionnels volontaires à se connecter sur une adresse Internet consacrée au questionnaire : ils pouvaient y répondre en plusieurs fois grâce à Lime Survey , logiciel d'enquête en ligne. Après trois mois, nous avons recueilli 418 questionnaires complets (soit $8 \%$ des mails envoyés), ce qui représente finalement un échantillon spontané de professionnels intéressés et volontaires pour s'exprimer sur la notion de bienveillance éducative. Ce tableau donne les types ${ }^{4}$ de professionnels qui ont répondu à l'enquête.

Figure 1 : Répartition des types de professionnels ayant répondu à l'enquête bienveillance

\begin{tabular}{|l|l|l|l|l|l|l|l|l|l|l|l|l|}
\hline PE & PLC & ASH & PLP & Form & CPE & Per-dir & Infir-scol & COP & AED & IEN & Adm & Total \\
\hline 74 & 191 & 26 & 31 & 16 & 26 & 23 & 7 & 4 & 4 & 1 & 15 & 418 \\
\hline $17,7 \%$ & $45,7 \%$ & $6,2 \%$ & $7,5 \%$ & $3,9 \%$ & $6,2 \%$ & $5,5 \%$ & $1,7 \%$ & $0,9 \%$ & $0,9 \%$ & $0,2 \%$ & $3,6 \%$ & $100 \%$ \\
\hline
\end{tabular}

es enseignants représentent au minimum 77 \% (322) du total des réponses car il y a 25 anciens enseignants parmi les formateurs (8), les inspecteurs (1), les CPE (1) et les personnels de direction (15), soit un total de 347 (83\%). Cette importante majorité laisse à penser que l'école bienveillante est essentiellement une préoccupation pédagogique d'enseignants.

10 Nous avons progressivement construit ce questionnaire à la suite de nos diverses lectures. Nous avons croisé l'analyse des prescriptions institutionnelles (cf. partie 1 précédente) à des travaux scientifiques sur des notions relativement proches de la bienveillance 
éducative, comme peu de recherches avaient été effectuées explicitement sur cet objet. Nous nous sommes d'abord intéressé aux théories du care (Gilligan, 1982; Laugier \& Paperman, 2006; Molinier, 2009, 2013; Tronto, 2009), ainsi qu'aux pratiques du caring (Noddings, 1984, 2012) afin de tisser des liens notionnels et praxéologiques avec la bienveillance éducative. Nous nous sommes appuyé sur le numéro thématique de la revue Éducation \& socialisation, Les cahiers du CERFEE: «Le care en éducation: quelle(s) reconfiguration(s)?» (2016). Nous avons croisé l'ensemble de ces lectures avec quelquesunes de nos références en sciences de l'éducation en matière de pédagogie (Altet, 2006; Houssaye, 1993 ; Meirieu, 1987, 2009) et de didactique (Astolfi, 1987, 1992, 2008). Ces ouvrages pourraient bien sûr être accompagnés de bien d'autres, mais ils ont été des repères pour nous dans notre première carrière d'enseignant spécialisé puis dans nos premiers pas dans la recherche en éducation. C'est cette épistémologie personnelle ${ }^{5}$ (Hofer, 2004 ; Crahay et Fagnant, 2007) qui nous a amené à concevoir cette enquête sur la bienveillance éducative, dans une perspective compréhensive, du point de vue des professionnels. Nous avons ensuite affiné notre questionnaire suite à la passation et à l'analyse de huit entretiens exploratoires semi-directifs : deux avec des personnels de direction, un avec un CPE, un avec un formateur d'enseignants, deux avec des professeurs des écoles (PE) et deux avec des professeurs de lycée ou collège (PLC). Ces entretiens exploratoires semi-directifs ont été menés autour de cinq questions (Qu'est-ce qu'une " école bienveillante » pour vous? Que pensez-vous des prescriptions autour de l'école bienveillante? Quels dispositifs ou quelles actions pourraient contribuer à construire une école bienveillante? Quels gestes professionnels bienveillants pourriez-vous décrire? Comment articuler bienveillance et exigence ?)

11 Le questionnaire débutait par le recueil d'informations préalables sur le profil professionnel des enquêtés, à travers cinq questions: poste actuel, ancienneté dans le poste et dans le métier, autres expériences dans l'éducation ou la formation, et quatre mots-clés des valeurs relatives à l'identité professionnelle défendue.

12 La première partie de l'enquête visait à appréhender les représentations des professionnels de l'école sur la notion de bienveillance. Les enquêtés devaient répondre à onze questions à choix multiples pour tenter de caractériser la bienveillance à l'école. Premièrement, ils devaient donner leur avis sur les prescriptions officielles qui souhaitent «conforter une école bienveillante» ("Que pensez-vous des textes officiels qui souhaitent consolider une école bienveillante?»). Ils devaient ensuite réfléchir au vécu de leurs élèves ( Pensez-vous que les élèves perçoivent l'école comme bienveillante?»). Conformément aux textes officiels qui relient bienveillance et exigence, les enquêtés devaient se positionner par rapport à la réalité de l'articulation entre bienveillance et exigence ( Que pensez-vous du lien entre bienveillance et exigence?»). Ils avaient ensuite à réfléchir et donner leur avis sur différentes associations (couples notionnels construits à la suite des lectures citées précédemment): bienveillance et climat scolaire ("Que pensez-vous du lien entre bienveillance et climat scolaire?»), bienveillance et motivation, bienveillance et autorité, bienveillance et sentiment de justice scolaire, bienveillance et empathie, bienveillance et lien école-famille, puis bienveillance et responsabilités institutionnelles. Pour clore cette première partie, la onzième question visait la caractérisation de la bienveillance à l'école : la question invitait les enquêtés à choisir trois expressions représentatives parmi douze (quatre positives, quatre neutres et quatre négatives). 
13 La seconde partie de l'enquête comportait neuf questions ouvertes pour une approche qualitative de la notion de bienveillance à l'école, afin d'appréhender les pratiques déclarées et les principales préoccupations des professionnels pour développer la bienveillance à l'école. La première série de cinq questions visait à comprendre comment s'exprime la bienveillance dans les relations interpersonnelles, avec les élèves, avec les familles, avec les collègues, avec la hiérarchie, et avec les autres partenaires de l'école. Toutes ces questions débutaient de la même façon : «Comment s'exprime la bienveillance dans vos relations professionnelles avec...? " La cinquième question proposait ensuite aux professionnels d'illustrer l'expression de la bienveillance dans leurs pratiques quotidiennes, sans autres précisions afin de laisser libre cours aux interprétations («Comment s'exprime la bienveillance dans vos pratiques professionnelles quotidiennes? »). La sixième question visait une dimension fondamentale dans les textes officiels: les pratiques d'évaluation qui se doivent d'être à la fois bienveillantes et exigeantes («Comment vos pratiques d'évaluation peuvent-elles être à la fois bienveillantes et exigeantes?»). La septième question interrogeait l'articulation globale entre bienveillance et exigence à l'école («Comment réussissez-vous à être exigeant avec les élèves tout en restant bienveillant?»). La huitième question proposait de s'intéresser aux responsabilités collectives de l'organisation scolaire qui pourraient permettre de développer la bienveillance à l'école ("Quelles organisations, dispositifs ou projets pourraient permettre de consolider une école bienveillante ?»). La neuvième et dernière question était une tribune ouverte laissée aux enquêtés afin qu'ils s'expriment librement sur la notion de bienveillance à l'école (« Vous pouvez apporter d'éventuelles précisions sur l'école bienveillante que le questionnaire n'aborderait pas »).

Il nous semble important de souligner que cette enquête repose exclusivement sur le volontariat, à partir d'un échantillon strictement aléatoire de $20 \%$ des personnels de l'académie de Caen sur l'année scolaire 2015-2016. Si l'échantillon de départ était représentatif de l'ensemble des personnels de l'académie, l'échantillon des 418 réponses complètes est constitué de personnes intéressées par l'enquête et motivées à prendre du temps pour y répondre. La plupart du temps ces professionnels semblaient convaincus de l'importance de la bienveillance à l'école, mais quelques-uns ont émis des points de vue plus nuancés et critiques, voire parfois hostiles à la notion de bienveillance, aux réformes pédagogiques associées et à l'intérêt même de cette enquête. Les résultats suivants sont donc à appréhender avec prudence. Si la seconde partie qualitative du questionnaire est plus ouverte aux descriptions libres, elle n'a été renseignée la plupart du temps que par des professionnels convaincus de l'importance de la bienveillance à l'école.

\section{Résultats de l'enquête sur la bienveillance à l'école}

\subsection{Approche des représentations des professionnels de l'Éducation nationale sur la bienveillance à l'école}

15 Nous présentons les résultats de la première partie de l'enquête sous forme de tableaux pour en faciliter la lecture question par question. Ces données chiffrées indiquent que les professionnels de l'école qui ont répondu à cette enquête s'inscrivent majoritairement dans les perspectives de la bienveillance éducative. Ils font le lien avec l'approche systémique sur le climat scolaire $(80,1 \%)$, la motivation $(83,4 \%)$, l'exigence $(83,5 \%)$, l'autorité éducative $(90,4 \%)$, le sentiment de justice scolaire $(82,3 \%)$, l'empathie $(85,1 \%)$ 
et le lien école-famille $(90,4 \%)$. Un tiers d'entre eux $(28,7 \%)$ considèrent que les élèves doivent globalement ressentir du stress à l'école, quand un sur dix $(9,5 \%)$ pensent plutôt que l'école est majoritairement perçue comme étant bienveillante. Ces résultats nous permettent d'attendre des réponses intéressantes dans la seconde partie de l'enquête, qui questionnera plus ouvertement les principales préoccupations et pratiques déclarées de ces professionnels de l'école convaincus de l'importance de la bienveillance éducative. Il faut noter que si une courte majorité $(55,7 \%)$ considère la bienveillance comme " fondamentale et conforme à leurs valeurs ", un tiers non négligeable (33,9\%) souligne qu'il s'agit d'une notion encore "trop floue ", ce qui légitime l'intérêt de cette étude visant à caractériser la bienveillance éducative du point de vue des professionnels de l'école, tout en conservant un regard critique, notamment sur les responsabilités institutionnelles qui ne sont pas à négliger. Les deux tiers (63,1\%) les pensent « importantes mais les équipes éducatives peuvent malgré tout consolider une école bienveillante » alors que $20 \%$ les appréhendent comme totalement responsables, quand $a$ contrario, $15,8 \%$ les considèrent «négligeables: seuls les acteurs de terrain sont responsables du climat scolaire».

Si les expressions plébiscitées pour caractériser la bienveillance à l'école sont relativement positives (1- «un facteur de réussite et de progrès » à $21,6 \%, 2$ - « une pédagogie positive ${ }^{6}$ » à 16,5 \% 3- « une condition nécessaire pour un bon climat scolaire " à 15,4\%, 4- « une posture éthique » à 12,5\%,5- « une attitude empathique indispensable » à $10,9 \%$ ), il ne faut pas occulter les craintes ou les doutes de certains professionnels ( « une nouvelle mode institutionnelle » à 3,9\%, « un concept mou mal défini » à 3,5\%, « une surresponsabilisation des équipes éducatives » à $2,1 \%$, « un cache-misère » à 1,8 \% ou " une dérive du pédagogisme » à $1,4 \%$ ) que nous questionnerons dans notre partie discussion.

\subsection{Approche des pratiques déclarées et des principales préoccupations des professionnels de l'Éducation nationale sur la bienveillance à l'école}

17 Dans une perspective descriptive et compréhensive, nous avons tenté de catégoriser les réponses des professionnels pour chacune des questions posées afin de mieux appréhender leurs principales pratiques déclarées et préoccupations pour conforter une école bienveillante.

\subsubsection{La bienveillance dans les relations avec les élèves et les familles}

Pour faciliter la lecture, nous avons choisi de regrouper ces deux premières questions. En effet, elles ont suscité chez les professionnels des réponses du même type, principalement basées sur la construction progressive d'un climat de confiance.

Les notions d'accueil et de disponibilité ont été largement évoquées dans les questions relatives aux relations aux élèves et à leurs familles. Que ce soit à la grille de l'établissement, dans la cour, à la porte de la salle de cours ou du bureau, la qualité de l'accueil est revendiquée pour beaucoup de professionnels de l'école interrogés, dans la diversité des fonctions occupées: enseignant, chef d'établissement, CPE, infirmière scolaire ou assistante sociale par exemple. Au-delà des réunions parents-professeurs organisées institutionnellement par l'établissement, il semble important d'offrir d'autres 
possibilités de rencontre qui puissent s'adapter aux situations professionnelles des parents dans la mesure du possible ( Je leur téléphone ou je corresponds avec eux par courriel si besoin », «j'ai contacté au téléphone les parents afin d'établir une relation de confiance »). S'adresser aux élèves en les appelant par leur prénom permet un accueil personnalisé selon certains enquêtés, et certains ajoutent même l'importance du sourire. Cette autorité souriante permettrait aux éducateurs d'articuler la qualité de la relation à l'exigence des attentes envers les élèves.

Sans surprise, l'écoute et le dialogue sont des attitudes professionnelles plébiscitées par les enquêtés, même si quelques enseignants disent qu'ils ne sont pas là pour «faire l'assistante sociale » et préfèrent déléguer à une collègue ou à l'infirmière scolaire les besoins de dialoguer des élèves. Cependant, l'écoute semble être la préoccupation centrale pour construire des relations de qualité avec les familles, comme ces verbatim l'illustrent: «je suis convaincu que pour les convaincre de quoi que ce soit, je dois d'abord savoir les écouter ». Une enseignante propose «une écoute sincère (je suis maman aussi) » pour «faire que les rencontres soient de réels échanges». Pour que le dialogue fonctionne, il faut «du respect, de l'humilité, du non-jugement», mais également être " pédagogue dans les propositions qui sont faites ». L'écoute des parents est primordiale pour "tenir compte de leur parole, de leur vision du monde scolaire", " de leurs angoisses » et "pour mieux comprendre leur enfant", toujours dans le souci d'instaurer un « climat de confiance réciproque ».

21 L'empathie s'articule étroitement avec les postures d'écoute et de dialogue pour tenter de comprendre les élèves : « les écouter pour comprendre leur problème avant de remédier à leurs difficultés ", développer " une écoute réelle des besoins de l'élève et un respect de sa personnalité » pour «entendre les difficultés réelles des élèves ». L'empathie pour comprendre les parents a été explicitement soulignée par certains enquêtés pour « être à l'écoute de la situation de chacun ", et "essayer de se mettre à la place des familles", pour " comprendre leur point de vue », et «l'organisation de la famille pour trouver un moyen d'aider l'élève en difficulté en dehors du collège». Certains tentent "de comprendre la perception de l'école par les familles », mais «ne juge[nt] pas l'éducation de l'élève, et essaye[nt] de faire un lien entre les codes familiaux et les codes de l'école ».

La communication est également largement évoquée, sous différentes formes, avec une attention particulière à ce qui est dit (ou écrit) aux élèves ou à leurs familles. Ces préoccupations situent encore une fois le langage comme un véritable instrument professionnel (Bucheton et al., 2004; Saillot, 2014), comme dans l'attention portée aux appréciations, annotations et remarques aux élèves ou à leurs familles. Cette vigilance des professionnels quant aux mots qu'ils emploient à l'écrit ou à l'oral pour décrire le travail et le comportement des élèves s'inscrit totalement dans cette recherche de bienveillance perçue par les élèves, ou a minima pour éviter tout sentiment de manque de bienveillance ou d'injustice scolaire. Un enseignant souligne que «les parents doivent percevoir que mon attitude envers leur enfant serait la même que j'adopterais avec mon enfant ", ce qui constitue un véritable principe éthique. Apporter des réponses objectives dans la bonne temporalité constitue une préoccupation relativement partagée. Certains professionnels souhaitent adopter une posture d'accompagnement (Paul, 2016) respectueuse du cheminement de leur interlocuteur avec quelques précautions : «pas donneur de leçon sur la parentalité ", " en associant les parents à la recherche de solutions », et «ne pas culpabiliser les parents, mais les responsabiliser ». 
23 La valorisation des efforts des élèves, de leurs réussites est l'une des préoccupations importantes des professionnels interrogés : réaliser des feed-back aux élèves (et à leurs familles) relatifs à l'évolution positive de leur engagement dans leur travail scolaire, et à l'acquisition de nouvelles connaissances et compétences. Ce type de préoccupations vise à changer positivement le regard des autres et de l'élève lui-même sur son propre travail afin de mettre en mots le postulat d'éducabilité («considérer tous les élèves comme capables de réussir ", "valoriser toutes les réussites, même les plus modestes", "en tenant compte de leur potentiel et de leur niveau de départ », « Les élèves doivent sentir que leur réussite m'importe.»). Un autre enseignant souligne que «les élèves doivent avoir confiance s'ils sentent que je crois en eux ». La bienveillance s'exprime ici par les encouragements pour «mener l'élève vers ce qu'il peut donner de meilleur, tant sur le plan intellectuel que sur le plan comportemental». Certains souhaitent «insister sur chacune des progressions" pour "leur montrer qu'ils sont capables de réussir». L'ensemble des gestes professionnels pour valoriser les élèves permet le renforcement de leur sentiment de compétences (Bandura, 2003) et "leur redonner confiance" pour reprendre les mots des enquêtés. Valoriser est donc également une préoccupation récurrente dans les relations avec les familles («Ne pas mettre d'étiquette sur leur enfant, en ne portant pas de jugement sur ce qu'il est (ou nous semble être)»), avec parfois des réalités difficiles («Certaines familles sont peu bienveillantes avec leurs enfants. Il nous faut souvent féliciter les élèves devant leur famille pour qu'ils prennent confiance en eux »).

Veiller au sentiment de justice scolaire est une préoccupation fondamentale pour certains enquêtés afin de construire une relation de confiance tant avec les élèves qu'avec leurs familles. Il semble nécessaire d'« être le plus juste possible envers chaque élève, et de les traiter avec impartialité ». Un autre professionnel déclare «essayer d'appliquer les sanctions de manière juste et équitable ». Ainsi l'équipe éducative doit mettre en place un «système clair de punition accepté par les élèves, avec une reprise des situations de conflits dans une ambiance apaisée ». Si la volonté récurrente d'«être juste» fait référence à la justice scolaire, un chef d'établissement précise que «la justesse est un élément indispensable ainsi que la transparence du discours et des actes posés, identiques et valables pour tous ». Encore une fois, si la bienveillance dans les échanges est mobilisée dans «le respect total de la justice et de l'équité dans le traitement des élèves », un « cadre clairement défini » doit dessiner les contours d'exigences indispensables à toute éducation. Cependant, une posture d'authenticité assez rogérienne est également illustrée: «Je sais reconnaître quand je me trompe et sais m'en excuser auprès des élèves ».

Certains témoignages ont insisté sur l'importance du regard qui donne confiance (Marcelli, 2006) : «Je pense que le regard d'une personne est essentiel. Il est porteur de l'épanouissement de l'autre. Il met en confiance et permet l'apprentissage. ». L'idée de "bien-être " est également évoquée, notamment par le "rire ensemble ", "l'humour " pour «faire en sorte que l'élève ait le sourire en entrant en cours... et en sortant aussi », qu'il « soit heureux d'être là ».

\subsubsection{La bienveillance dans les relations avec les collègues et les autres professionnels partenaires}

Pour les relations avec les collègues, il semble que ce soit finalement parfois plus difficile qu'il n'y parait car, parfois, la bienveillance semble « difficile avec des collègues rigides » 
ou « absente car certains collègues sont en souffrance » et « dans certains établissements, c'est chacun pour soi ». Malgré tout, la majorité des témoignages reçus se centre sur les principales préoccupations suivantes : le dialogue et l'écoute, la confiance dans le respect des pratiques de chacun ( $\mathrm{Ne}$ pas dénigrer le travail des collègues» est primordial, comme « le respect du point de vue de chacun »), l'attente d'un engagement mutuel pour construire « un esprit d'équipe » et "des projets communs", la disponibilité en cas de besoin et l'entraide sont également plébiscitées (« rendre service » aux collègues et «se serrer les coudes »), sans oublier la convivialité : "bonne humeur", "gâteaux, café partagés ", «blagues et petites histoires drôles », pour « rire ensemble » et même «bien vivre ensemble ».

27 Pour les relations avec les autres professionnels partenaires, les préoccupations précédentes reviennent avec quelques nuances. La disponibilité pour " prendre le temps de se rencontrer " avec "courtoisie», "cordialité », "ponctualité », dans une forme récurrente de politesse souriante. L'écoute et le dialogue pour se concerter, parfois «se décentrer de l'école", avec "curiosité ", "réel intérêt », et "respect mutuel des professionnalités de chacun ». Cette « reconnaissance du travail des partenaires » semble fondamentale, «sans jugement ", mais pour " valoriser le rôle de chacun » en arrivant à comprendre les contraintes des uns et des autres pour leur «donner les moyens de travailler ».

\subsubsection{La bienveillance dans les relations avec la hiérarchie}

Tout d'abord, nous avons regroupé les témoignages de l'absence de bienveillance de la part de la hiérarchie ressentie par les professionnels de l'école qui n'ont "pas l'impression de bienveillance de leur part». Les demandes de bienveillance seraient "toujours dans le même sens" car les personnels ont souvent "l'impression de ne pas être écoutés » et « la hiérarchie ne montre pas du tout l'exemple. On doit faire preuve de bienveillance auprès des élèves mais les inspections en manquent souvent ».

Les témoignages de marques de bienveillance de la part de la hiérarchie ressenties par les professionnels sont plus rares mais existent dans notre enquête. Un professionnel dit "s'appuyer sur la hiérarchie lorsqu'il rencontre une difficulté ", quand un autre pense que " ses supérieurs sont à l'écoute de ses difficultés ». Un enseignant considère que son inspecteur "est une personne bienveillante qui a toujours le souci de notre bien-être ", quand un autre décrit recevoir un "soutien moral ou organisationnel» par "des échanges réguliers et une confiance pour les initiatives». L'un d'entre eux se réjouit "d'avoir une hiérarchie compréhensive actuellement, ce qui n'a pas toujours été le cas ».

Certaines propositions apportent des pistes pour développer des relations bienveillantes avec la hiérarchie, comme « une loyauté non exempte de suggestions et d'une lecture critique au service des élèves", une "confiance professionnelle réciproque », une " disponibilité des deux côtés ", "tenant compte des contraintes de chacun ", «l'écoute et le dialogue » et parfois même "un droit à l'erreur ». Il s'agirait de "considérer les supérieurs plutôt comme des partenaires que des ennemis", dans une approche « respectueuse et loyale».

31 La reconnaissance et l'institutionnalisation des temps de concertation des enseignants apparaissent indispensables pour prévenir et limiter leur souffrance (Lantheaume \& Hélou, 2008). Les personnels «croulent sous les tâches administratives de plus en plus lourdes » et les "conditions de travail se dégradent régulièrement ». Cette situation 
critique jouerait « en défaveur de la bienveillance des personnels à cause du stress et du manque de sérénité ». Certains enseignants ont également souligné leurs besoins en matière de formations qui ne trouvent pas toujours des réponses dans les plans de formations proposés.

Les relations avec la hiérarchie semblent parfois parasitées par certaines décisions institutionnelles qui jouent un rôle central pour beaucoup d'enquêtés qui ressentent une forme d'empêchement ou d'obstacles grandissants pour construire une école bienveillante (fermetures de classes ou d'établissements, dotations horaires globales qui ont des conséquences sur les effectifs des classes, la mise en place de dispositifs d'accompagnement des élèves, et autres projets pédagogiques innovants).

\subsubsection{La bienveillance dans les pratiques d'évaluation et de différenciation}

33 Cette question a fait l'objet de nombreuses propositions, ce qui montre que les professionnels de l'école qui ont répondu sont convaincus que les pratiques d'évaluation et de différenciation sont au cœur de « l'école bienveillante et exigeante ».

34 Sans parler explicitement d'empathie cognitive, les enseignants cherchent à "comprendre ce que les élèves ne comprennent pas", et "pourquoi ils sont en difficultés ", ce qui revient à essayer de "comprendre la démarche intellectuelle de l'élève » pour reprendre des verbatim de l'enquête. Un enseignant spécialisé indique qu'il « individualise la progression pédagogique pour chaque élève, évalue selon l'acquisition de compétences, et adapte les tâches demandées aux élèves de façon différenciée ». Un autre souhaite « l'accompagnement des enseignants vers une pédagogie de la réussite, qui se préoccupe de n'exclure aucun élève, ce qui génère un enseignement soucieux de tous ». Un enseignant veille à "apporter son soutien et ses conseils pour solutionner leurs difficultés » et un autre fait "attention aux temporalités et au potentiel de chacun ». Certains préconisent la pratique de la «métacognition pour faire prendre conscience aux élèves de leur profil et améliorer leurs façons d'apprendre ». Des enseignants souhaitent avoir des moments en dehors des cours collectifs pour « favoriser les échanges et mieux individualiser les exercices, sans avoir à gérer l'ensemble du groupe classe ». L'approche par compétences permettrait à certains de mieux personnaliser leurs conseils et leurs groupes de travail en cours, en réalisant parfois avec les élèves " un contrat de progrès " avec des « objectifs adaptés à chaque élève ». Il s'agit de prendre « en compte leur niveau réel pour les mettre en situation de progrès » et non pas leur niveau supposé, ce qui génère parfois du décrochage scolaire. Un enseignant nous alerte sur l'importance de "proposer une pédagogie différenciée mais ambitieuse », car parfois pédagogie de la réussite a rimé avec baisse des exigences (« Toujours exiger le meilleur de chacun à l'aune de ses capacités. »).

Ces préoccupations autour des pratiques différenciées et individualisées passent obligatoirement par une réflexion sur la dimension formatrice aux erreurs et le statut donné aux évaluations. Un enseignant résume l'essentiel: «Dédramatiser l'erreur (en expliquant qu'on apprend en se trompant, cela fait partie du jeu et est indispensable pour progresser), dédramatiser les évaluations (l'important c'est de progresser, de comprendre ce qui n'a pas été).». Au lieu de pointer les erreurs comme des fautes ou des manques anormaux, il s'agit de «s'appuyer dessus pour mieux conseiller les élèves». Un enseignant déclare " proposer un contrôle de rattrapage quand un contrôle n'a pas été réussi ou valoriser la correction de l'élève par des points bonus ", ce qui valorise la 
persévérance si importante dans les travaux de Viau (1994) sur la motivation en contexte scolaire. Vingt ans après l'ouvrage de référence d'Astolfi (1997), l'erreur peut-elle être réellement appréhendée par l'enseignant comme "un outil pour enseigner » et par l'élève comme un outil pour mieux apprendre et progresser? Certains enseignants ont souligné l'importance des critères d'évaluation explicites et compris de tous " pour qu'ils apprennent à s'auto-évaluer avant même de recevoir leurs notes. Ainsi le sentiment d'injustice est fortement amoindri ». Ces préoccupations s'inscrivent dans la perspective d'un contrat de confiance (Antibi, 2014).

\section{Analyse et discussion autour de la notion d'école bienveillante}

Nous pouvons à présent construire un réseau de préoccupations qui permettrait de " conforter une école bienveillante et exigeante ", du point de vue des professionnels de l'éducation qui ont répondu à cette enquête. Leurs propositions mettent en lumière un registre assez homogène de postures et d'attitudes professionnelles relativement transversales aux différentes fonctions des professionnels, basées sur l'accueil et la disponibilité, l'écoute empathique et le dialogue constructif, la reconnaissance et la valorisation, avec des boussoles éthiques orientées vers la confiance, la justice, et l'éducabilité. D'autres préoccupations sont centrées autour de gestes professionnels reliés plus étroitement aux questions d'apprentissages : les pratiques d'évaluation formatrice et de différenciation pédagogique pour tenter de s'ajuster (Bucheton \& Soulé, 2009) aux besoins des élèves en considérant l'erreur comme un outil pour enseigner (Astolfi, 1997). Si ces quelques lignes définissent la bienveillance dans le champ scolaire à la suite de cette enquête, certains pourraient sans doute y ajouter d'autres registres professionnels qui leur tiennent à cœur comme les pédagogies coopératives (Connac, 2009). En effet, chaque professionnel définit la bienveillance selon son épistémologie pratique ${ }^{7}$ (Sensevy, 2007) et son vécu.

Au-delà du projet scientifique de caractérisation de la bienveillance éducative, cette enquête a également permis de poser quelques éléments de discussion plus critiques sur cette notion qui relève pour certains d'un simple effet de mode. Nous avons donc pris en compte les craintes et les critiques des enquêtés, notamment dans la dernière question "tribune libre", mais également les questionnements et controverses soulevés lors des débats du symposium sur la bienveillance éducative que nous avons coordonné à Caen les 19 et 20 octobre 2017 dans le cadre du colloque « Enjeux, débats et perspectives : 50 ans des sciences de l'éducation ».

Si cette enquête a permis d'éclaircir la notion d'école bienveillante par la caractérisation de préoccupations et de gestes professionnels partagés mieux identifiés, elle a également souligné en creux certaines craintes, limites ou ambiguïtés.

Premièrement, les principales critiques questionnent l'authenticité des intentions prescriptives autour de l'école bienveillante. Certains alertent sur une éventuelle dérive laxiste. Ils ont «l'impression que la bienveillance est la nouvelle excuse pour justifier n'importe quel comportement d'élève » ou craignent de « tomber dans le pédagogisme et le laxisme, en oubliant que l'exigence est une obligation éducative pour un adolescent qui manque de repères ». D'autres craignent que l'école bienveillante soit une sorte d'élixir pour panser les maux de l'école, en dissimulant parfois des apprentissages insuffisants, 
notamment avec un «système de notation au brevet des collèges [qui] permet d'augmenter artificiellement les résultats alors que les acquis des élèves semblent de plus en plus fragiles ».

40 Deuxièmement, l'autre critique serait que les prescriptions pour une école bienveillante feraient le procès plus ou moins implicite des enseignants malveillants. Déplacer le débat sur le terrain de la malveillance intentionnelle ne permet pas de réfléchir sereinement aux enjeux éducatifs attendus, même s'il ne faut pas ignorer les travaux de Merle (2005) qui mettent en lumière certaines humiliations d'élèves à l'école, ni les approches cliniques de la relation d'enseignement qui analysent certains comportements d'enseignants comme relevant de formes inconscientes de "sadisme ordinaire bien tempéré » (Blanchard-Laville, 2011). Au lieu de cristalliser les discussions autour d'une hypothétique malveillance intentionnelle des enseignants, la focale pourrait être déplacée sur un éventuel manque de bienveillance parfois ressenti par les élèves. Certains personnels interrogés soulignent que «trop d'enseignants laissent à penser à certains élèves qu'ils ne sont pas capables de progresser ".

41 Troisièmement, les prescriptions pour une école bienveillante n'apporteraient rien de nouveau. La plupart des enseignants ne les ont pas attendues. Ces injonctions créent parfois des réactions de rejet de principe alors que les intentions pédagogiques sont similaires : «Je ne me réfère pas à cette notion. J'essaie d'être juste et rigoureuse dans mes exigences. Je respecte les élèves et j'essaie d'être juste avec eux ». Pour certains, il ne s'agirait que d'une question de bon sens. Cependant, en croisant les données recueillies, il ne semble pas que ce bon sens soit totalement partagé dans les équipes et incarné dans des postures professionnelles communes. Les personnels qui disent défendre les principes éthiques relatifs à l'essence même de l'éducation, comme l'éducabilité ou la liberté, adoptent des positions parfois diamétralement opposées aux injonctions à l'école bienveillante. Soit ils considèrent que la bienveillance est une approche complètement cohérente avec ces principes, soit ils considèrent qu'elle n'apporte rien de nouveau et qu'elle n'est qu'une sorte de « fourre-tout » pédagogique.

Une quatrième critique dénonce une nouvelle stratégie managériale, hypothèse corroborée par les travaux de Linhart (2015) qui considère ce type d'approche prétendument plus humaniste comme une véritable imposture pour permettre d'augmenter insidieusement la pression sur les personnels dans une application stricte et exacerbée du taylorisme. Dans le cadre de l'Éducation nationale, l'argument de la bienveillance fait peser sur les épaules des professionnels des réalités scolaires peu reluisantes tout en essayant de faire oublier les responsabilités institutionnelles. Les prescriptions à conforter une école bienveillante sont-elles une solution pédagogique ou une simple rhétorique pour répondre aux mauvais résultats des évaluations internationales (Les enquêtes Pisa de l'OCDE situent la France comme l'un des pays les plus inégalitaires en matière d'apprentissages scolaires)?

Cinquièmement, la bienveillance serait essentiellement une affaire d'attitude mobilisant des "savoir-faire discrets» comme pour le care (Molinier, 2009). L'exemple le plus emblématique de notre enquête serait l'autorité souriante décrite par les professionnels. La bienveillance serait « ce sourire de l'œil » (Nietzsche, 1878). L'importance du sourire et du regard souligne des dimensions très difficiles à appréhender méthodologiquement en recherche mais dont l'authenticité semble une des clés de relations interindividuelles sereines. Le neurobiologiste Duchenne de Boulogne a montré dans ses travaux de la fin du $\mathrm{XIX}^{\mathrm{e}}$ siècle que les sourires sincères sont accompagnés de la contraction du muscle 
orbiculaire de l'œil, ce qui a été confirmé par Ekman (1990) avec l'appellation « sourire de Duchenne». Cependant, suffit-il de sourire, d'avoir l'air sympathique pour être bienveillant? S'agit-il finalement de bienveillance ou de bientraitance ? L'une des clés de la réponse se situerait dans les intentions de la personne, conformes ou non aux principes éthiques de l'éducation énoncés plus haut, avec toute la difficulté pour le chercheur d'accéder à ces données.

Les récents travaux de Prairat (2017) sur le tact pourraient apporter des pistes de réflexion intéressantes pour donner davantage de consistance aux intentions et aux gestes professionnels que les enseignants mettent en œuvre et aux principes éthiques qui les sous-tendent.

Sixièmement, la bienveillance évoquée par certains professionnels pour caractériser leurs relations avec les familles ne glisse-t-elle pas parfois vers une forme de paternalisme ? En effet, si certains disent se baser sur l'écoute attentive et l'empathie pour accompagner chemin faisant (Paul, 2016) les familles, d'autres se placent en experts qui informent les parents par un discours de vérité, les conseillent " pour le bien de l'élève » car ils savent « ce qui est bien pour [lui] ». Mais le bien de l'élève est-il toujours univoque et facilement identifiable? De plus, est-ce à l'enseignant seul de déterminer le bien de l'élève ? La bienveillance pourrait être évoquée de façon abusive par certains professionnels qui auraient peut-être égaré la boussole éthique pour problématiser des questions éducatives complexes (Fabre, 2011).

\section{Conclusion}

Ce type d'enquête auprès des professionnels de l'Éducation nationale sur la bienveillance était inédit en France au moment de son lancement. Parallèlement, et sans concertation avant 2017, une recherche doctorale a été menée par Reto (2018) avec des résultats relativement proches au niveau des «invariants qui composent la bienveillance ». Les principales préoccupations des professionnels interrogés pour conforter une école bienveillante et exigeante résident dans des classes de situations et des postures professionnelles caractéristiques. La bienveillance à l'école s'exprimerait chez les professionnels concernés par l'importance des notions d'accueil et de disponibilité, le développement de postures d'écoute qui favorisent le dialogue et l'empathie, la réflexion autour d'un agir communicationnel (Habermas, 1987) qui permette de valoriser les efforts et les réussites des élèves, tout en restant exigeant tant au niveau du cadre éducatif que des apprentissages, dans le souci permanent de veiller au sentiment de justice scolaire.

Au-delà des critiques et des réserves vis-à-vis de la place de la bienveillance dans les prescriptions ou dans les discours politiques, nous pensons que c'est la dichotomie bienveillance-exigence qui incarne l'essence même de l'école bienveillante, voire de l'école tout court tant elle incarne les mêmes contradictions inhérentes à l'éducation ou les mêmes dilemmes pédagogiques (Meirieu, 1987). Selon un enquêté, «l'exigence est une forme de respect, au contraire de l'absence d'exigence qui est une forme de mépris qui signifierait implicitement que l'élève ne serait pas capable de progresser ». Pour un autre professionnel, « l'exigence c'est avoir de l'ambition pour chaque élève ». Il semble que le challenge de la dichotomie bienveillance-exigence réside dans l'élaboration d'une pédagogie différenciée qui permette de "refuser les dénivelés d'exigence » (Terrail, 2016, p. 76). Pour certains, la bienveillance permettrait un cercle vertueux : « La bienveillance 
permet d'être exigeant». "Lorsque mon attitude est bienveillante, je me sens plus légitime pour élever mon niveau d'exigence envers les élèves ».

Un enquêté nous invite à ne pas oublier que la bienveillance, «c'est surtout donner le plaisir d'apprendre ». Réussir à articuler exigence et bienveillance permettrait de réunir la «saveur des savoirs" (Astolfi, 2008) et le plaisir d'apprendre pour les élèves. Cette dichotomie bienveillance-exigence semble au cœur des préoccupations des professionnels de l'école en matière d'évaluation et de différenciation pédagogique. Comment réussir à s'ajuster aux besoins spécifiques des élèves en conservant un haut niveau d'exigence ? $\mathrm{Ce}$ dilemme était déjà au centre des préoccupations pédagogiques qui tentaient il y a quelques années non pas de conforter une école bienveillante mais de développer une "pédagogie de la réussite ». Cette dernière expression avait le mérite de souligner une dimension fondamentale : "s'intéresser aux apprentissages des élèves c'est cela la vraie bienveillance» (Bautier, 2014), en conservant un haut niveau d'exigence adapté au potentiel de chaque élève dans la perspective d'un école inclusive (Gardou, 2012; Tremblay, 2012).

\section{BIBLIOGRAPHIE}

Altet, M. (2006). Les pédagogies de l'apprentissage. Paris : PUF.

Amigues, R. (2009). Le travail enseignant : prescriptions et dimensions collectives de l'activité. Les Sciences de l'Éducation pour l'Ère Nouvelle, 42(2), 11-26.

Antibi, A. (2014). Pour des élèves heureux en travaillant ou les bienfaits de l'évaluation par contrat de confiance. Toulouse : Éditions Math'adore.

Astolfi, J.-P. (1992). L'école pour apprendre. Paris : ESF.

Astolfi, J-P. (1997). L'erreur, un outil pour enseigner., Paris : ESF.

Astolfi, J.-P. (2008). La saveur des savoirs. Disciplines et plaisir d'apprendre., Paris : ESF.

Bandura, A. (2003). Auto-efficacité, le sentiment d'efficacité personnelle. Bruxelles : De Boeck.

Bautier, E. (2014). L'évaluation par compétences peut laisser de côté des choses fondamentales. Café pédagogique. Repéré à : http://www.cafepedagogique.net/lexpresso/

Pages/2014/12/15122014Article635542258733620673.aspx (Consulté le 13 septembre 2017).

Blanchard-Laville, C. (2011/1). Pour un accompagnement clinique groupal du travail enseignant. Nouvelle revue de psychosociologie, 11, 131-147.

Bucheton, D., Bronner, A., Broussal, D., Jorro, A. \& Largier, M. (2004). Les pratiques langagières des enseignants : des savoirs professionnels inédits en formation, Repères, 30, 33-53.

Bucheton, D. \& Soulé, Y. (2009). Les gestes professionnels et le jeu des postures de l'enseignant dans la classe : un multi-agenda de préoccupations enchâssées, Éducation et didactique, 3, 3.

Connac, S. (2009). Apprendre avec les pédagogies coopératives : démarches et outils pour l'école. Paris : ESF. 
Crahay, M. \& Fagnant, A. (2007). À propos de l'épistémologie personnelle : un état des recherches anglo-saxonnes. Revue française de pédagogie, 161, 79-117.

Curie, J. \& Dupuy, R. (1996). L'organisation du travail contre l'unité du travailleur, dans Y. Clot (dir.). Les histoires de la psychologie du travail (pp. 179-194), Toulouse : Octarès.

Ekman, P., Davidson, R. J., \& Friesen, W. V. (1990). The Duchenne smile : emotional expression and brain physiology. II. Journal of personality and social psychology, 58 (2), 342-353.

Fabre, M. (2011). Éduquer pour un monde problématique. La carte et la boussole. Paris : PUF.

Faïta, D. \& Saujat, F. (2010). Développer l'activité des enseignants pour comprendre et transformer leur travail : un cadre théorique et méthodologique. Dans F. Yvon et F. Saussez (éds.), Analyser l'activité enseignante : des outils méthodologiques et théoriques pour l'intervention et la formation (pp. 41-71). Québec : Presses universitaires de Laval.

Gardou, C. (2012). La société inclusive, parlons-en! Il n'y a pas de vie minuscule. Toulouse : Éditions Érès.

Gilligan, C. (1982). In a Different Voice. Psychological theory and women. Cambridge : Harvard University Press.

Habermas, J. (1987) Théorie de l'agir communicationnel. Rationalité de l'agir et rationalisation de la société (Tome 1). Paris : Fayard.

Hofer, B. K. (2004). Epistemological understanding as a metacognitive process: Thinking aloud during online searching. Educational Psychologist, 29(1), 43-55.

Houssaye, J. (dir.), (1993). La pédagogie, une encyclopédie pour aujourd'hui. Paris : ESF.

Lantheaume, F. \& Hélou, C. (2008). La souffrance des enseignants. Une sociologie pragmatique du travail enseignant. Paris : PUF.

Lapeyronnie, D. (2014). Synthèse des travaux du Conseil national de l'innovation pour la réussite éducative 2013-2014. Paris : Ministère de l'Éducation nationale, de l'Enseignement supérieur et de la Recherche.

Laugier, S. \& Paperman, P. (dir.) (2006). Le Souci des autres. Éthique et politique du care. Paris : EHESS.

Linhart, D. (2015). La comédie humaine du travail. De la déshumanisation taylorienne à la surhumanisation managériale. Toulouse : Éditions Érès, coll. « Sociologie clinique ».

Marcelli, D. (2006). Les yeux dans les yeux. L'énigme du regard. Paris : Albin Michel.

Meirieu, P. (1987). Apprendre... Oui, mais comment. Paris : ESF.

Meirieu, P. (2009). Le pari de l'éducabilité. Les soirées de l'ENPJJ, Les cahiers dynamiques, 43.

Merle, P. (2005). L'élève humilié. L'école, un espace de non-droit? Paris : PUF.

Molinier, P. (2009). Temps professionnel et temps personnel des travailleuses du care : perméabilité ou clivage ?, Temporalités, 9. Repéré à : http://temporalites.revues.org/988 (Consulté le 3 septembre 2017).

Molinier, P. (2013). Le travail du care. Paris : La Dispute.

Nietzsche, F. (1878). Humains, trop humains (première partie, trad. par A.-M. Desrousseaux). Paris : Société du Mercure de France.

Noddings, N. (1984). Caring, a Feminine Approach to Ethics and Moral Education. Berkeley : University of California Press. 
Nodding, N. (2012). The caring relation in teaching. Oxford Review of Education, XXXVIII(6), 771-781.

Nunziati, G. (1990) Pour construire un dispositif d'évaluation formatrice, Cahiers pédagogiques, 280, 47-64.

Paul, M. (2016). La démarche d'accompagnement, Repères méthodologiques et ressources théoriques. Bruxelles : De Boeck Supérieur.

Prairat, E. (2017). Éduquer avec tact, Vertu et compétence de l'enseignant. Paris : ESF, coll. « Sciences humaines ».

Reto, G. (2018). La bienveillance dans le champ scolaire. Caractérisation des pratiques et actualisation selon des membres du personnel enseignant de collège, des chefs d'établissement et des experts du monde de l'éducation. (Thèse de doctorat, soutenue à Angers le 29 juin 2018).

Saillot, E. (2014). Analyse des pratiques langagières d'explicitation des enseignants : études de cas en aide personnalisée à l'école primaire. Questions vives, recherches en éducation (Aix-Marseille), 21 (dossier thématique « Le travail collectif des enseignants en question »).

Saillot, E. (2015). Analyse des pratiques d'étayage des professeurs des écoles en situation d'aide personnalisée : modélisation d'une posture professionnelle, Les dossiers des Sciences de l'éducation, 34.

Sensevy, G. (2007). Des catégories pour décrire et comprendre l'action du professeur. Dans G. Sensevy \& A. Mercier (dir.), Agir ensemble : l'action didactique conjointe du professeur et des élèves. Rennes : PUR.

Tremblay, P. (2012). Inclusion scolaire, dispositifs et pratiques pédagogiques, Bruxelles : De Boeck Éducation.

Tronto, J. (2009). Un monde vulnérable. Pour une politique du care. Paris : Éditions La Découverte.

Viau, R. (1994). La motivation en contexte scolaire. Bruxelles : De Boeck Université.

\section{NOTES}

1. Loi d'orientation et de programmation pour la refondation de l'école de la République $\mathrm{n}^{\circ}$ 2013-595 du 13 juillet 2013.

2. Le site Éduscol «Climat scolaire» (www.cndp.fr/climatscolaire) propose des ressources théoriques et pratiques, issues d'une étroite collaboration entre des chercheurs et Caroline Veltcheff, ancienne déléguée ministérielle et actuelle directrice régionale de Canopé Normandie.

3. https://www.reseau-canope.fr/education-prioritaire/agir/priorite/conforter-une-ecolebienveillante-et-exigeante.html.

4. PE : professeurs des écoles; PLC : professeurs des lycées et collèges; ASH : enseignants spécialisés ; PLP : professeurs de lycées professionnels ; Form : formateurs d'enseignants ; CPE : conseillers principaux d'éducation ; Per-dir : personnels de direction des collèges et lycées ; infirscol: infirmières scolaires; COP : conseillers d'orientation psychologues; AED : assistants d'éducation en collèges ou lycées; IEN : Inspecteur $1^{\text {er }}$ degré ; Adm : personnels administratifs (secrétariat, gestion, collèges lycées)

5. Hofer (2004) appréhende l'épistémologie personnelle comme un ensemble de connaissances et de croyances se structurant progressivement en théories par des processus métacognitifs.

6. L'expression «pédagogie positive " n'est pas une notion structurée scientifiquement mais elle avait été citée par des professionnels lors des entretiens exploratoires, faisant un lien avec l'évaluation formatrice (Nunziati, 1990) et bienveillante qui appréhende l'erreur non pas comme 
une faute mais comme une étape de l'apprentissage et un véritable outil pour enseigner (Astolfi, 1997).

7. Sensevy (2007, pp. 37-38) définit l'épistémologie pratique comme l'ensemble des théorisations implicites ou explicites à propos des savoirs en jeu, leur enseignement et leur apprentissage.

\section{RÉSUMÉS}

La notion de bienveillance a émergé institutionnellement en France depuis la Loi de refondation de l'école de 2013. Cet article rend compte des principaux résultats d'une enquête réalisée en 2016 dans l'académie de Caen sur les représentations, les préoccupations et les pratiques déclarées des professionnels de l'école à partir des 418 questionnaires intégralement renseignés en ligne. Il s'agit d'essayer de mieux comprendre et définir la bienveillance éducative du point de vue des professionnels, en conservant un regard critique sur l'emploi de ce terme.

Caring school has been institutionally emerging in France since the school refoundation Law (2013). This text presents the main results of a survey realized in 2016 in the academy of Caen in Normandy. This study aims at describing and understanding school professionals' representations, concerns and self-reported practice, thanks to 418 full questionnaires. This text will finally take a critical look at caring and demanding school.

\section{INDEX}

Mots-clés : bienveillance éducative, école, enquête, représentations, préoccupations

Keywords : caring school, survey, representations, concerns

\section{AUTEUR}

\section{ÉRIC SAILLOT}

Maître de conférences en sciences de l'éducation, Université de Caen Normandie (CIRNEF) 